\title{
Regionalización dimensional del espacio social de la marginación municipal para el estado de Tamaulipas. México'
}

\author{
Dimensional regionalization of social environment \\ related to municipal marginalization; \\ in the State of Tamaulipas. México \\ Regionalizaçao dimensional do espaço social \\ da marginação municipal para o \\ estado de Tamaulipas. México.
}

Eder Jesús Noda Ramírez ${ }^{2}$ Adolfo Rogelio Cogco Calderón ${ }^{3}$ Universidad Autónoma de Tamaulipas

\section{Resumen}

El presente documento hace referencia a la regionalización del fenómeno de la marginación de acuerdo a sus dimensiones socioeconómicas para los municipios del estado de Tamaulipas, México, mediante la aplicación metodológica del análisis cluster jerárquico y bajo el criterio del espacio social de Bourdieu. Lo anterior tiene como finalidad hacer una combinación técnica de herramientas de la estadística multivariable con esquemas de regionalización socioespacial del fenómeno de la marginación para la relocalización de las carencias convergentes a la falta de acceso al desarrollo económico y a una mejor instrumentalización de la política social regional.

Palabras claves: marginación, regionalización, espacio social, política social, Tamaulipas.

I El presente documento se derivó del proyecto: Diagnóstico y análisis socioeconómico de las localidades y municipios de alta marginación en el Estado de Tamaulipas, Convenio CONACYT-Gobierno del Estado de Tamaulipas No. 107.213.

2 Lic. en Economía de Unidad Académica de Ciencias Jurídicas y Sociales (UACJS), Universidad Autónoma de Tamaulipas (UAT). Correo electrónico: eder.noda@gmail.com.

3 Doctor en Filosofía con Orientación al Trabajo Social y Políticas Comparadas del Bienestar Social. Profesor-Investigador de UACJS de la Universidad Autónoma de Tamaulipas. Miembro del Sistema Nacional de Investigadores. Correo electrónico: acogco@uat.edu.mx. 


\begin{abstract}
This document talks about the regionalization of marginalization phenomenon, based on the socioeconomic approach affecting municipalities in the state of Tamaulipas, México, by means of applying hierarchical cluster analysis methodology and as per Bourdieu's Social Space criterium. The aforementioned is aimed to technically combine tools of multivariable statistics together with socio-spatial regionalization patterns about the marginalization phenomenon for relocation of shortages dealing with lack of access to economic development and a better implementation of regional social policies.
\end{abstract}

Keywords: Marginalization, Regionalization, Social Space, Social Policy, Tamaulipas

\title{
Resumo
}

Este documento refere-se à regionalização do fenômeno da marginalidade com base em suas dimensões sócio-econômicas para os municípios no estado de Tamaulipas, no México, através da aplicação de metodologia de análise hierárquica de cluster, a critério do espaço social de Bourdieu.A intenção é fazer uma combinação de técnicas multivariadas ferramentas estatísticas para os padrões de regionalização sócio-espacial do fenômeno da marginalização para a relocalização das lacunas convergindo para a falta de acesso ao desenvolvimento econômico e uma melhor manipulação da política social regional.

Palavras-chave: Marginalização, a Regionalização, o Espaço Social, Política Social, Tamaulipas

\section{Introducción}

El estudio de la marginación como fenómeno socio espacial exige considerarla desde una perspectiva regional o cada vez más local, debido a que el espacio global si bien constituye un parámetro objetivo de la política social como eje fundamental del fomento al desarrollo y bienestar social. El reconocer al espacio local significaría una mejor instrumentalización de apoyo regional para el logro de esos objetivos comunes, derivados desde el centro y bajo un modelo de desarrollo económico y social incluyente.

En términos macroeconómicos, cuando la marginación pasa de la comprensión a su manifestación carencial en la población, inmediatamente el Estado se involucra como mediador de los desequilibrios sistémicos que traen consigo descoyunturas regionales, esto lo realiza a través del gasto público, lo cual deriva en toda una programación política para la atención social a espacios en mayores condiciones de marginación.

La marginación vista como un fenómeno estructural en un tiempo determinado, requiere de su concreción a través de indicadores de medición que sirvan a la determinación de políticas públicas basadas en la geografía primaria de las carencias básicas. 
Desde que se emprendió la tarea de medir la marginación por parte del Consejo Nacional de Población y Vivienda (CONAPO), la técnica utilizada siempre ha sido la de componentes principales, que resume una información global (a nivel nacional) para posteriormente distribuirla en cada uno de los diferentes espacios que componen el territorio mexicano.

Por casi 20 años, este indicador ha sido una herramienta indispensable para la distribución del ingreso en materia de lo social, así como en la realización de políticas públicas para el combate a la pobreza, la desigualdad y otras problemáticas socioeconómicas. Es decir, los grados de marginación inciden en la priorización de la política social en todos los niveles de gobierno.

Así pues, los espacios de mayor marginación recibirán mayores transferencias gubernamentales, por el contrario, a los de menor marginación se les asignará un presupuesto público en esa proporción.

Cabe mencionar también que la visión del CONAPO, por su característica institucional, es medir la marginación en una distribución del espacio político (estados, municipios y localidades) de acuerdo a indicadores sociales y económicos, lo que se traduce en resultados políticos asociados a determinados objetivos del Estado para ajustar su gasto público en los programas sociales y así cumplir con el discurso de equidad, lo que permitirá modificar la geografía de la marginación en un nivel cada vez más bajo, siendo esto un reflejo del progreso y desarrollo económico de la nación.

Para poder integrarse nuevamente a la función económica, la población desplazada por los conflictos estructurales del sistema económico es respaldada por el Estado. La política social se convierte en un instrumento indispensable para disminuir la marginación en las regiones y los países subdesarrollados y desarrollados.

El presupuesto público estará orientado a la protección social de los menos favorecidos en el desarrollo, reestructurando toda la política económica y fiscal para cubrir todas las prioridades que el Estado tiene, de tal forma que un aumento de la población en condición marginada presionará el aumento del gasto público debido a que su combate está relacionado con la educación, la vivienda, los salarios (empleo) y la salud.

La importancia de realizar ejercicios que permitan analizar de manera diferente a la marginación desde un punto de vista técnico reside en los planteamientos de índole estadístico y espacial del fenómeno, justo donde se inserta a esta lógica la discusión teórica del espacio social.

Ahora bien, la problemática consiste en responder a la cuestión de la espacialidad social del fenómeno de la marginación y la reconfiguración de sus espacios, y con base a ello hacer una mejor instrumentalización de la política social a nivel regional.

En este trabajo se considera como un no-espacio al individuo, ya que un ente aislado no conforma el espacio por su falta de relación con otros objetos de su entorno, por lo que se considera espacios representados a partir de aglomeraciones municipales de acuerdo a intensidades de exclusión social.

Lo anterior concuerda con lo discutido sobre el espacio social de Bourdieu algo que se irá explicando a lo largo del presente documento-, en donde la marginación se traslada a las microrregiones desarrolladas debido a que su gestación surge en los 
desequilibrios y procesos del desarrollo cada vez más concentrado en espacios más pequeños, formados, por un lado, por grupos de individuos regularmente vulnerables y también, por otra parte, a nivel macrorregional.

Esta concepción se traslada a la reconfiguración del espacio geográfico por uno social construido a partir de variables que definen la fisonomía territorial, como se verá en los siguientes párrafos. Esta investigación hará hincapié en el carácter macrorregional del espacio social a nivel municipal.

La estructura de este estudio consiste en primeramente hacer un breve marco conceptual de la marginación como fenómeno estructural y algunas de sus diferenciaciones sustanciales con otros problemas sociales como la marginalidad y la exclusión social. Luego, se abordará lo relacionado al espacio social y su vinculación con la marginación, haciendo una síntesis creativa. Por último, se presentará el desarrollo de la metodología para la determinación de las regiones y la reconfiguración del espacio por municipios similares en diferenciaciones o carencias socioeconómicas por cada dimensión de la marginación, la cual se analizará detenidamente.

\section{Sobre la marginación}

El punto de partida en el estudio de la marginación ha sido definir de manera clara y precisa el concepto. Definir este término es algo complicado, porque se ha convertido en un punto conexo de diferentes elementos estructurales y coyunturales analizados desde múltiples corrientes teóricas, lo que complica un concepto certero de marginación.

Cabe señalar que como el estudio de esta materia ha sido retomado sobre todo por economistas y sociólogos, las aseveraciones en torno a su definición varían ligeramente debido al enfoque o tipo de estudio que se le dé.

Campoy (2002) explica algunos enfoques de la marginación de acuerdo a realidades muy particulares. Se considerada pertinente mencionarlos, debido a que son versiones ya muy estudiadas en las últimas décadas y no son solo conjeturas filosóficas e ideológicas.

La primera tesis se refiere a la marginación como "un aspecto puntual y transitorio de la migración”. Por ejemplo: "un marginado sería un campesino todavía no integrado a la clase obrera urbana que, antes o después, terminará absorbiéndolo" (Campoy, 2002, p. I I). De ahí que las barriadas (espacio pobre) de las grandes ciudades, habitadas por migrantes de las áreas rurales, sean asociadas con el fenómeno de la marginación.

La autora refiere que esta concepción urbanística no define claramente la marginalidad puesto que la asocia con las barriadas y no considera el desarrollo histórico de esos lugares, como por ejemplo, las personas que nacen dentro de los barrios no tendrían denominación marginal al no ser migrantes.

Otro enfoque se deriva de la tesis desarrollista, principalmente expone que la pobreza y la marginación son efectos de las diferentes contradicciones del crecimiento económico auspiciado en un sistema capitalista excluyente debido a los siguientes 
factores: las insuficiencias del desarrollo agrícola, industrial y tecnológico, junto a la escasez de mano de obra cualificada y las desventajas del comercio exterior, lo que desencadena conflictos estructurales.

La polarización es una de las características principales de esta postura. Las contradicciones del modo de producción, basado en la acumulación del capital, son elementos inherentes a la evolución de todas las estructuras, de tal forma que el desarrollo y subdesarrollo son etapas de los procesos de adaptación y evolución del sistema capitalista, por lo que no pueden ser concebidos de forma aislada ambos conceptos. Derivado de lo anterior, la marginación es entonces:

(...) una manifestación dinámica de las sociedades en transición, de sus dualidades, asimetrías y discontinuidades: entre el artesanado y la gran industria; entre la vida parroquial de las aldeas atrasadas y las regiones metropolizadas modernas; entre las clases más pobres y menos educadas y las élites cosmopolitas de esas sociedades, etc. (Campoy, 2002, p. II).

La tercera tesis considera a la población marginal como "un problema de excedente demográfico (...), dicha consecuencia, se genera por la imposibilidad de insertarse en el sistema de producción, especialmente si se trata de sistemas capitalistas dependientes" (Campoy, 2002, p. I I). También considera otros elementos causales como el político, el religioso y el étnico.

La última concepción teórica en torno a la marginación y la pobreza considera los procesos de rechazo o la estigmatización social (teoría del etiquetaje), de ahí que su principio sea el concepto de "la diferencia", ya que es la principal causante de la exclusión de grupos marginados y pobres.

La marginación es una especie de penalización tanto social como jurídica, algo muy parecido a la discriminación; de tal forma que "es observada su existencia en todas las épocas y sociedades, el denominador común resulta ser la diferenciación, explicitada sociológicamente en estratificación y económicamente en desigual distribución de la riqueza" (Campoy, 2002, p. I2).

De acuerdo a las clasificaciones teóricas acerca de la marginación que expone Campoy, la más utilizada en la presente investigación así como en la mayoría de los estudios aquí mencionados es la relacionada con la tesis desarrollista debido a que es la más explorada y la que ha aportado el mayor número de elementos al análisis de este fenómeno multidimensional y estructural.

Con base en lo expuesto hasta el momento sobre la marginación, se puede observar la existencia de un arduo debate entre lo subjetivo y lo objetivo.

La subjetividad en la marginación está relacionada con las cuestiones psicosociológicas, culturalistas y filosóficas, en donde las diferenciaciones, las representaciones y las reproducciones sociales son los elementos que permiten definir una marginación de dimensiones abstractas e inclusive cualitativas y ubicadas en el paradigma de la modernización y de las sociedades post-modernas (Noda, 20l0). 
Lo objetivo en la marginación comprende una serie de elementos económicos y políticos de orden cuantitativos, sobre todo desde la consideración de factores asociados al desarrollo, cambio tecnológico, evolución histórica, comercio internacional, crecimiento económico, desarrollo regional, empleo, fuerzas productivas y relaciones sociales de producción, mismos que constituyen la comprensión concreta de la marginación y los fenómenos relacionados con ella como las desigualdades sociales y regionales, la pobreza y el atraso tecnológico, por citar a algunos (Noda, 20l0).

Ahora bien, conviene mencionar algunas definiciones ubicadas en la concepción objetiva de la marginación ya que son las más utilizadas en el presente trabajo, aunque sus aplicaciones convergerán desde una perspectiva sociológica y política. Específicamente se trabajará con lo analizado por el CONAPO, quien es el referente de conceptualización y medición de la marginación, al menos en México.

Una de las definiciones más utilizadas es la de Otero, quien comenta que la marginación social puede concebirse como un fenómeno espacial estructural expresado por las desigualdades respecto a las condiciones medias de vida, por lo que concierne a aspectos de índole socioeconómico (Otero, 2003).

Por otra parte, para Galvis la marginación no debe entenderse solamente por la carencia de ingresos (elemento también cuantitativo), pobreza relativa o de condiciones naturales, sino más bien "como una función carencial del marco necesario para que la región potencialice las capacidades y competencias regionales en un ámbito nacional e inclusive mundial” (Galvis, 2002, p. 91); también es una condición de exclusión de un espacio regional al no articularse a las condiciones del desarrollo.

De acuerdo con la Coordinación General del Plan Nacional de Zonas Deprimidas y Grupos Marginados COPLAMAR (1977, 1979, I98I), la marginación se utilizaría para "caracterizar aquellos grupos que han quedado al margen de los beneficios del desarrollo nacional y de los beneficios de la riqueza generada, pero no necesariamente al margen de la generación de riqueza ni mucho menos de las condiciones que la hacen posible" (Coplamar, 1979, XII), de ahí que espacialmente se ubicara a la marginación en lo rural y lo urbano.

Luego, el CONAPO $(1993,1999)$ sintetizó de manera creativa el concepto, al afirmar que "la marginación social puede ser entendida como fenómeno estructural múltiple, que integra en una sola valoración las distintas dimensiones, formas e intensidades de exclusión o no participación en el proceso de desarrollo y en el disfrute de sus beneficios" (Conapo-Cna, 1993, p. 5).

Esta última definición expresa de forma más acertada y concreta la conceptualización de la marginación, contemplada desde un escenario multidimensional en función de las diferentes maneras e intensidades de exclusión.

El elemento de privación hacia los beneficios del desarrollo económico, menciona Cogco (2010), manifiesta prácticamente el sentido de la marginación, considerándose entonces un fenómeno social carencial o deficitario, es decir, el concepto en sí mismo implica la dificultad de integración al desarrollo. 
Como puede observarse, la concepción del CONAPO sobre la marginación tiene su origen en la teoría desarrollista, estructuralista y objetiva; aunque más recientemente la corriente de la sociología y psicología moderna ha generado una concepción más holística y, a su vez, subjetiva del tema, sobre todo después de la reforma económica de la mayoría de los países latinoamericanos.

Sin embargo, aunque muchas investigaciones continúen bajo esta línea, varios de los trabajos mencionados en esta investigación reconocen los conceptos y metodología del CONAPO, quien se ha convertido en la versión oficial del fenómeno estructural de la marginación en el país, pero aún no generalizada en todo Latinoamérica.

Como se puede apreciar, la temática de la marginación está aún inconclusa y con el paso del tiempo ha incorporado nuevas interpretaciones en aquellos conceptos que, en un inicio, fueron considerados como sinónimos para señalar a la marginación como un fenómeno social.

Conceptos como marginalidad, exclusión, vulnerabilidad y pobreza fueron considerados conceptos que se remitían a la situación de personas que viven en condiciones no equitativas o similares a las del resto de la población que tiene acceso a los beneficios proporcionados por el desarrollo.

Una de las precisiones que recientemente se han hecho al concepto de marginación es la aportación de Cortés (2002) quien hace la diferenciación entre la marginación y la marginalidad; en el primer caso se refiere al fenómeno estructural espacial con un carácter territorial, centrándose en las localidades, municipios y estados, en tanto que la marginalidad es un concepto que alude a los aspectos de índole personal, a los sujetos que viven desde su individualidad, factores tanto físicos como psicológicos de exclusión, que los hacen sentirse fuera de los beneficios que el desarrollo proporciona a las localidades o municipios de carácter urbano.

Cortés parte de las herramientas metodológicas de Bunge, específicamente en el desarrollo de los conceptos que se entienden por "unidades de significado", éstos pueden ser exactos o vagos, aplicables o inaplicables. El complemento o predicado del concepto pueden ser "unarios (trabaja), binarios (se relaciona), ternario (se interpone), cuaternario (intercambia), etc.” (Cortés, 2006, p. 5). Explica también cómo un predicado unario se refiere solamente al individuo, espacio o relación social.

De acuerdo con la lógica de Bunge, la clase de referencia unaria del predicado debe atender a la integración o "colección" de los objetos. Cortés cita ejemplos basados en esta extensión metodológica del concepto: "La marginalidad predica sobre individuos y la marginación sobre entidades geográficas” (Cortés, 2006, p. 4).

Por otra parte, la noción de marginalidad incluye a la marginación, pues la primera contiene las mismas dimensiones conceptuales que la segunda, sin embargo el concepto de marginalidad considera además aspectos sociopsicológicos, culturales y políticos (Cogco, 2010). Esta diferenciación entre marginalidad y marginación ha sido muy creativa y da una posible respuesta a todas las discusiones teóricas sobre el concepto, conciliando a las dos visiones teórico-prácticas. 
En resumen la marginación como fenómeno social, económico y espacial ha tenido diferentes acepciones que pueden notarse en trabajos de índole estructuralistas básicamente en Latinoamérica.

Estas concepciones tanto objetivas como subjetivas han preponderado las principales discusiones teóricas sobre dicha temática dándole características cada vez más específicas que le han permitido diferenciarse de conceptos como marginalidad y exclusión social, que al relacionarse con el desarrollo local y la importancia que ha tenido el Estado por medio de su intervención, su comprensión regional ofrece una mayor perspectiva local de este fenómeno.

Es importante relacionar la comprensión de la marginación con la del espacio social desde la óptica de Bourdieu, la cual se mencionará en los siguientes párrafos.

\section{El espacio social y la marginación socio-espacial}

Continuando con el análisis teórico de la marginación como fenómeno socio espacial, se expondrán en las siguientes líneas y en forma de reflexión, las discusiones sobre esto desarrollado por Pierre Bourdieu,y se asociarán con la lógica del discurso teórico que se ha mencionado hasta el momento.

Esta concepción teórica tiene un enfoque muy diferente respecto a la mirada economista, debido a su naturaleza meramente sociológica, lo que converge en un análisis holístico que permite realizar generalizaciones y categorías.

La marginación desde la perspectiva regional se traslada a todos los espacios y forma micro y macro localizaciones espaciales que indican las carencias de la población, mismas que impiden disfrutar del desarrollo, así también, dichas carencias muestran una patología de algún tipo de pobreza. En el espacio social, esta localización se distingue por las "diferenciaciones" de los grupos individuos, aunque se desconoce el límite del espacio al que pueda trascender este fenómeno estructural (Noda, 2010).

Existe una dinámica en las reproducciones sociales de grupos e individuos cada vez más autónoma, lo que genera una dispersión colectiva afectada por situaciones exógenas como las tensiones entre las fuerzas del mercado y las relaciones sociales de producción.

Esta dispersión de las reproducciones sociales pueden darse entre regiones desarrolladas y subdesarrolladas a tal grado de llegar al espacio social de la marginación ya sea macro o micro e inclusive formando otro tipo de regiones en función de las distinciones sociales, las cuales se convierten en el eje principal de la formación socio espacial, a diferencia del sistema carencial en una lógica económica.

Las relaciones entre grupos e individuos manifiestan la realidad de una sociedad distribuida en una geografía específica, lo que se convierte en un entorno multidimensional donde cada agente social (colectivo o individual) interactúa de manera diferente en función de determinada sectorización social (Bourdieu, 1993).

Entonces "el espacio social es una construcción que, evidentemente, no es igual al espacio geográfico: define acercamientos y distancias sociales” (Gutiérrez, 
2003, p. 34). Esta definición señala bien la importancia geográfica de los agentes, pero sobre todo la importancia del desenvolvimiento social de estos actores en tal geografía definida. De tal forma que los grupos sociales determinarán consciente $o$ inconscientemente los límites espaciales que caracterizan una identidad regional, pero con base en las presiones sistémicas del libre del mercado, por lo que todo espacio se adecúa a la dinámica global de los diferentes actores estructurales.

La marginación como fenómeno socio-espacial comienza a ser abordada desde la perspectiva sociológica antes que económica, debido a que la primera perspectiva analiza a la sociedad en el punto de que los desequilibrios regionales económicos se trasladan hasta un espacio no precisamente económico.

En detalle hace una radiografía de la diferenciación como producto de lo relacional y real. Si bien estas diferenciaciones son producto de una contradicción del capitalismo, el hecho social de que sea un fenómeno reproductivo y variante de la sociedad y de su entorno, convierte a este análisis en una herramienta fundamental para continuar comprendiendo la espacialidad de la marginación y sus respectivas regionalizaciones desde una reconfiguración del espacio social y sus aplicaciones al espacio político-geográfico.

Bourdieu comienza su análisis original con una distinción y argumenta que la diferencia entre los sujetos y objetos conforma una relación que explica la existencia misma, es decir, las desviaciones sociales son reales sólo porque son relacionales. Explica que, en esencia, la diferencia:

Fundamenta la noción misma de espacio, conjunto de posiciones distintas o coexistentes, externas unas a otras, definidas en relación unas de otras, por su exterioridad mutua y por relaciones de proximidad de vecindad o de alejamiento y asimismo correlaciones de orden, como por encima, por debajo y entre (Bourdieu, 2003, p. 16).

En síntesis, la geografía del espacio social se trazará a partir de la distinción en una sociedad, tal distinción será determinada por el "espacio de toma de decisiones" y se reflejará en los patrones de conducta de las sociedades, inclusive en el mínimo espacio, siempre y cuando exista al menos algún tipo de "desviaciones diferenciales". La base de la diferenciación, de acuerdo al autor, será el capital cultural y económico, factores que han sido utilizados de manera eficiente por los países desarrollados.

(...) El espacio social se constituye de tal forma que los agentes o los grupos se distribuyen en él, en función de su posición en las distribuciones estadísticas, según los dos principios de diferenciación, que, en las sociedades más avanzadas, como EUA, Japón y Francia, son sin duda los más eficientes, el capital económico y el capital cultural. (Bourdieu, 2003, p. 16). 
La idea del aprovechamiento del espacio social se refiere a que la percepción hacia el otro constituye un tipo de relación causal en los arquetipos y las representaciones sociales de una población localizada en un territorio dado.

El capital económico y cultural de las naciones desarrolladas en contraste con el de los países no desarrollados establece un patrón de conducta y transformación del mismo espacio, donde los signos y símbolos constituyen otros órdenes sociales (familia, Estado, instituciones, entre otros) precisamente a través de las diferenciaciones sociales simbólicas (paradigmas, lenguaje, representaciones, entre otros). Lo anterior afecta a todas las reproducciones sociales, siendo entonces la "significación" de la realidad un elemento muy asociado al espacio social y simbólico.

Por ejemplo, la estética de la clase dominante se impone sobre la población civilizada, a diferencia de la clase dominada. Otro ejemplo, es cómo los intelectuales tienden a votar más por partidos de ideología política de izquierda, y los empresarios por partidos políticos de ideología derechista, habiendo determinando así un tipo de espacio social, relacional y causal (personalidades del oficio-ideología política).

Ahora bien, en lo que respecta a la marginación, las diferenciaciones sociales determinadas por los que sí tienen viviendas con acceso al servicio de luz eléctrica en contraste con los que no tienen, representan un orden social diferente dentro de un mismo espacio, que dicha segregación reproduce un espacio distinto entre los que cuentan o no con dicho servicio público, afectando toda la dinámica de interacción social con sus respectivos símbolos y significados. Esta lógica se traslada ahora a los espacios que vienen a ser los diferentes municipios que conforman al estado.

Respecto a las clases sociales, Bourdieu reconoce que no se puede negarlas bajo un criterio conservador, tampoco se pueden comprobar desde un enfoque crítico.Al respecto, incluso critica a Marx, refiriéndose a las refutaciones que éste le hacía a Hegel sobre "llevar a cabo un salto mortal de la existencia en teoría a la existencia en práctica” (Bourdieu, 2003, p. 23), el autor explica que la visión teórica de Marx sobre la resistencia de los grupos sociales determina un pensamiento de clase, por lo tanto este pensamiento repercute en las percepciones de la praxis, cayendo el mismo Marx en la debilidad ontológica de la dialéctica hegeliana, puesto que las clases sociales no existen en teoría, lo que existe es el espacio social:

La existencia de clases, en teoría y sobre todo en la realidad, es, como todos sabemos por experiencia, una apuesta de luchas. $Y$ ahí reside el obstáculo principal para un conocimiento científico del mundo social y para la solución (porque hay una...) del problema de las clases sociales. Negar la existencia de las clases, como se ha desempeñado en hacerlo la tradición conservadora en nombre de unos argumentos que no son todos ni siempre absurdos, es en última instancia negar la existencia de diferencias, y de principios de diferenciación (Bourdieu, 2003: 24). 
Las clases sociales no existen (aun cuando la labor política orientada por la teoría de Marx haya podido distribuir, en algunos casos, a hacerlas existir por lo menos a través de las instancias de movilización y de los mandatarios). Lo que existe es un espacio social, un espacio de diferencias, en el que las clases existen en cierto modo en estado virtual, en punteado, no como algo dado sino como algo que se trata de construir (Bourdieu, 2003, p. 24.).

Un proceso inverso al de la diferenciación supone la proximidad en el espacio social, lo que provoca la reducción del mismo, es decir, entre los grupos sociales existen puntos de coincidencia que acortan distancias culturales y económicas; sin embargo, de acuerdo a las categorías de Bourdieu, esto no significa que exista una clase determinada puesto que las reproducciones sociales nunca llegan a ser las mismas, sobre todo desde la percepción de las cosas o los objetos.

La marginación, bajo la lógica de Bourdieu, es una diferenciación del desarrollo, bienestar e integración social y económica de las regiones y se convierte en el polo opuesto de estos aspectos, asimismo los desequilibrios regionales se dan por el tipo de interacciones sociales.

La marginación regional del espacio social se genera en cualquier dinámica de las reproducciones y de cohesión; no es que exista una dualidad de clase, como lo mencionan los neomarxistas y estructuralistas, puesto que de acuerdo a este análisis, no existen tales clases, lo que hay es el espacio social.

Entonces el fenómeno de la marginación regional se convierte en uno de los tantos espacios sociales que existen en la realidad significativa y diferencial, nótese que el desarrollo económico es también una realidad específica. Las distinciones socioeconómicas se concretan en aquellos indicadores de exclusión social al representar distancias y proximidades tanto de grupos como de regiones.

Cabe mencionar que los "marginados" y los "integrados" recrean subespacios y subsistemas en el espacio social, es decir, aparecen microrregiones, puesto que internamente sus interacciones son diferentes, por las mismas condiciones regionales, estructurales y circunstanciales que le rodean, cayendo en una lógica de las diferenciaciones de las diferenciaciones, pero que en un término común existe una proximidad.

Un ejemplo de lo mencionado es el de la población que cuenta con acceso al servicio de luz eléctrica. Es posible que los que "no cuentan" con electricidad, pese a que bajo ese criterio determinen un espacio social muy particular, en ese mismo grupo existan otros significados y percepciones de otras cosas tales como la participación ciudadana o las preferencias electorales, aunque no dejan de mantener patrones similares, condicionales y conductuales como la pobreza o la representación social de la siembra.

Otra aplicación desde el enfoque de Bourdieu es que permite acoplar las diferenciaciones a cualquier multinivel, es decir, que va más allá de un límite espacial convencional (geográfico) dejando abierta la posibilidad de la incorporación de diferentes concepciones sociales de la realidad actual sobre el fenómeno de la marginación, ahora 
bajo el contexto de la modernidad y la globalización y las complejas dinámicas estructurales entre países desarrollados y subdesarrollados.

Cabe reflexionar acerca de cuál es el límite espacial de la marginación regional; al menos visto desde la lógica de Bourdieu, sería la desintegración del espacio social, es decir, cuando deje de existir lo real y lo relacional entre las diferenciaciones, algo que en toda la historia racional del hombre no ha ocurrido. La marginación desde la óptica del espacio social del autor, marca una diferencia en las relaciones o interacciones dentro del sistema de producción imperante.

Lo anterior, motiva a una discusión actual de la espacialidad de la marginación en función del esquema de los espacios sociales, en donde no existen las clases y las aproximaciones no son más que la sectorización del mismo espacio.

Por lo tanto, se reconoce a través de esta investigación que la marginación se traslada a cualquier región desarrollada y subdesarrollada de las sociedades diferenciadas, muy posiblemente representada en indicadores polarizados, tal como lo ha hecho el CONAPO por medio de la "estructura carencial del desarrollo", llamada desde una perspectiva más reflexiva y sociológica "estructura diferencial del desarrollo", aunque en su ontología difieren, debido a que la segunda implica la inserción del capital cultural y los límites espaciales son más amplios y siempre existentes por los procesos "relacionales" a diferencia de la primera.

\section{Metodología para la regionalización de la marginación para Tamaulipas}

En este apartado se describirá la metodología planteada para regionalizar componentes de la marginación en el estado de Tamaulipas a nivel municipal con base a las dimensiones socioeconómicas de la marginación. El objetivo es identificar la relación socio espacial en cada uno de los elementos que componen el fenómeno y así vislumbrar el acomodamiento de la geografía del espacio social con base en esta problemática.

La composición del índice de marginación consiste en cuatro dimensiones socioeconómicas y nueve variables de exclusión social, las cuales construirán de forma separada los diferentes espacios sociales del fenómeno de la marginación para apoyo de la política social municipal.

El siguiente esquema, muestra de forma gráfica la composición estructural de la marginación: 
Figura I. Dimensiones socioeconómicas y formas de exclusión social del fenómeno estructural y multidimensional de la marginación.

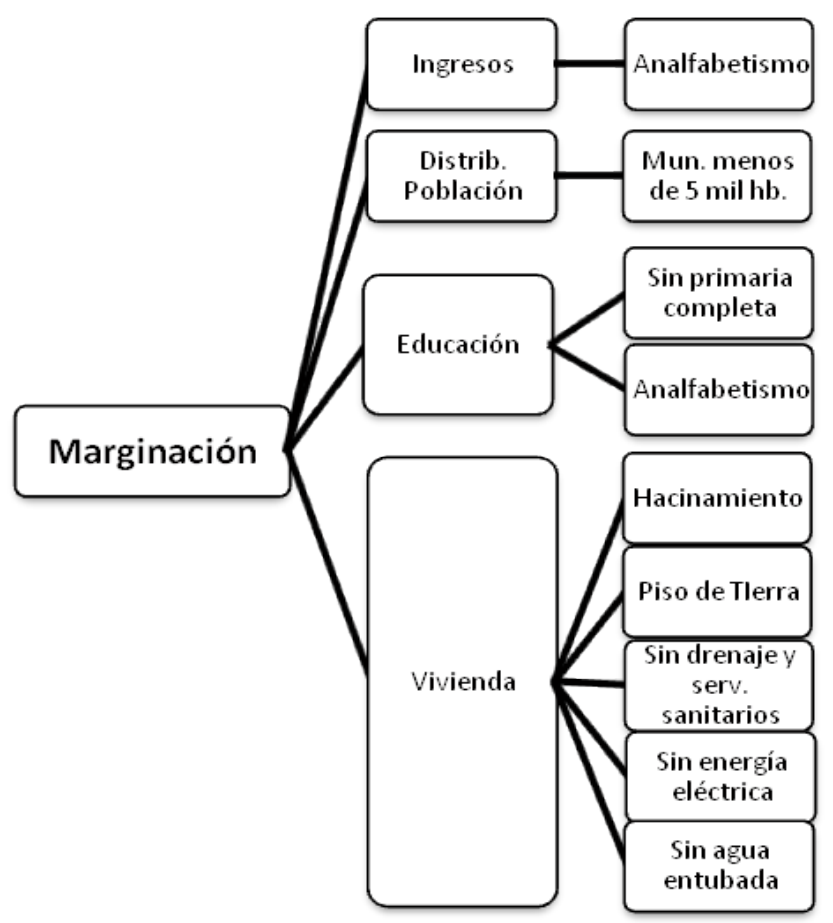

Fuente: CONAPO, 2005.

Actualmente ${ }^{4}$, el estado de Tamaulipas está compuesto por 43 municipios, los cuales se clasifican de acuerdo a su índice de marginación en: I 3 de muy baja marginación, 7 de baja, 17 de media y 6 de alta marginación. Lo que demuestra zonas prioritarias en materia de política social, como es el caso de los municipios de alta marginación.

Para identificar las diferentes formas de exclusión es necesario construir espacios que presenten características similares u homogéneas, así pues, cada dimensión socioeconómica compone cuatro grandes grupos, distribuidos de acuerdo al indicador correspondiente de cada concepto.

Este tipo de análisis requiere la aplicación de modelos jerárquicos. La técnica cluster análisis sirve "para clasificar un conjunto de individuos -localidades- en una serie de grupos-espacios” (Visauta y Martori, 2003, p. 178). Por lo que la inserción de la perspectiva regional respecto a la geografía social de la marginación se adecua a esta técnica de agrupación de espacios. 
Para continuar con la formulación de un modelo integral de la marginación, basado en grupos socioeconómicos, interesa definir las diferentes distancias, en términos euclidianos, para establecer una matriz de proximidad entre las diferentes variables de criterio, correspondientes a las formas de exclusión:

$$
d^{2}(x, y)=\sum_{i}\left(x_{i}-y_{i}\right)^{2}
$$

Donde $d$ es la distancia euclidiana elevada al cuadrado y $X$ e $Y$ son las variables de exclusión representadas en una matriz propia de los i-ésimos espacios de los conglomerados jerárquicos.

Una vez calculada la matriz de distancia, se procede a la aplicación del método de vinculación inter-grupos, el cual consiste en definir de la manera más aproximada la distancia de todas las combinaciones posibles entre dos conglomerados o clusters, aprovechando la información de los pares de dichas distancias, lo que logrará homogeneizar el espacio, es decir, conocer los dos elementos más próximos (Visauta y Martori, 2003):

$$
d_{A B}=\frac{1}{n_{A} n_{B}} \sum_{i=A} \sum_{i=B} d_{i j}
$$

Donde son las diferentes combinaciones de distancias de los clusters bidimensionales en los determinados espacios.

Una ventaja de los indicadores que componen el índice de marginación es que se encuentran en términos porcentuales, lo que permite continuar con una igualdad de unidades. Después de calculadas las distancias inter-grupos de los espacios por dimensión socioeconómica, se analizan los números de grupos definidos de acuerdo a su dendograma. ${ }^{5}$

Como se pretende regionalizar el espacio único e integral (espacio político), se procedió al establecimiento de clusters que representaran significativamente la distribución de los municipios de Tamaulipas, así que se analizó la posibilidad de determinarlos por el criterio de la re-escalada de sus distancias ( 0 a 25), sin embargo, como se pretende obtener un número reducido de clusters adaptados a una regionalización factible, se tomó la re-escalada más larga, ya que el proceso inverso indicaría un aumento de grupos.

Para fines prácticos, se procedió a definir cinco grupos según los niveles de aglomeración por dimensión socioeconómica:

$$
E_{i k j k}=e_{i 1} e_{j 1}, e_{i 2} e_{j 2} \ldots e_{i k j k}
$$

5 El dendograma es la representación gráfica que facilita la interpretación de un cluster cualquiera. Su presentación es en forma de árbol, resumiendo el proceso de agrupación del análisis cluster. La conexión entre enlaces similares se determina por el nivel de similitud o disimilitud entre grupos.Véase a VicenteVillardón en: <http://benjamindespensa. tripod.com/spss/AC.pdf> y a Marcial Terrádez en: <www.uoc.edu/in3/emath/docs/Cluster.pdf> 
Donde $E$ es el grupo integral definido por las dimensiones socioeconómicas (4), i es el número de municipios dentro del grupo, considerando valores de I a 43, j son las variables de exclusión y $\mathrm{k}$ el número de inter-grupos definidos (5) que especifican espacialmente la dimensión socioeconómica. ${ }^{6}$

Geográficamente, la división política del estado de Tamaulipas constituye el espacio integral $(E)$ que a su vez se reconfigura en los espacios sociales de la marginación (el...4) en función de la regionalización de las dimensiones socioeconómicas de ésta.

Figura 2. Espacio geográfico (división política municipal) del estado de Tamaulipas. ${ }^{7}$

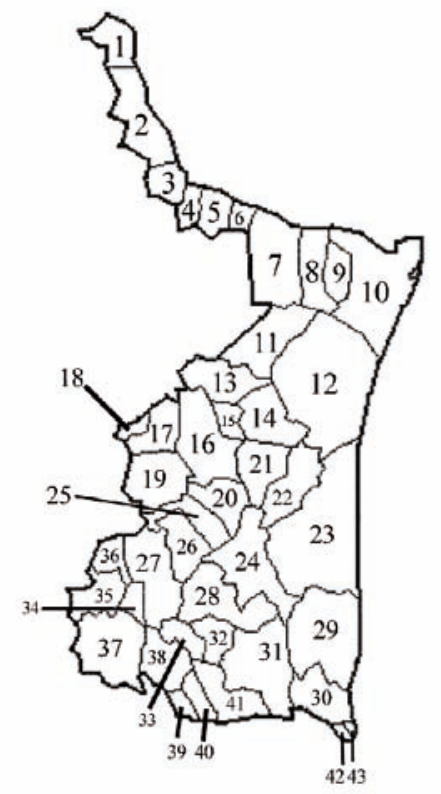

1 Nuevo Laredo
2 Guerrero
3 Mier
4 Miguel Alemán
5 Camargo
6 Gustavo Diaz Ordáz
7 Reynosa
8 Río Bravo
9 Valle Hermoso
10 Matamoros
11 Mendez
12 San Fernando
13 Burgos
14 Cruillas
15 San Nicolás
16 San Carlos
17 Villagrán
18 Mainero
19 Hidalgo
20 Padilla
21 Jiménez
22 Abasolo
23 Soto la Marina
24Casas
25 Gómez

26 Victoria
27 Jaumave
28 Llera
29 Aldama
30 Altamira
31 González
32 Xicoténcatl
33 Gómez Farias
34 Palmillas
35 Bustamente
36 Miquihuana
37 Tula
38 Ocampo
39 Nuevo Morelos
40 Antiguo Morelos
41 Mante
42 Tampico
43 Madero

43 Madero

Fuente: Elaboración propia.

6 Desde una perspectiva geográfica, el espacio integral (E) es la división política del estado de Tamaulipas, y las otras cuatro regiones $\left(e_{1} \ldots{ }_{4}\right)$ corresponden al espacio social de las dimensiones socioeconómicas de la marginación.

7 El estado de Tamaulipas se encuentra en la parte norte de la República mexicana. Colindante al sur con los estados de Veracruz y San Luis Potosí; al este, con el Golfo de México; al oeste con el estado de Nuevo León; y, al norte con el estado de Texas perteneciente a los Estados Unidos. Su superficie abarca el 4,1\% del territorio nacional, distribuida en 43 municipios.

Para el 2005 su densidad poblacional se aproximaba a los tres millones de habitantes, representando el 2,9\% de todo el país.

Sus principales actividades económicas son el comercio, el turismo y la industria, sumando un $44,6 \%$ de Producto Interno Bruto (PIB) a nivel estatal; con respecto al PIB nacional participa con un 3,2\% siendo la onceava economía más importante del país. Véase el Instituto Nacional de Estadística, Geografía e Informática en: <http://cuentame. inegi.org.mx/monografias/informacion/tam/>. 
De esta manera quedan definidos los grupos homogéneos que determinan la clasificación de la marginación y se establece el modelo regional que incluye las diferentes regiones por dimensión socioeconómica y las variables de exclusión de los espacios geográficos municipales de Tamaulipas.

Se considera que las dimensiones socioeconómicas aglomeradas en el espacio hacen referencia a las carencias potenciales del desarrollo y a las diferencias sociales bajo una perspectiva regional, propios de la geografía del espacio social de los municipios del estado de Tamaulipas.

En este caso, el modelo de regionalización de indicadores no se basa en el modelo factorial; si bien el índice de marginación es una medida sintética que deriva en un espacio ortonormal, todos los componentes de la marginación para después regionalizar los espacios “i” por estratos que denoten los niveles de marginación.

El modelo de aglomeración jerárquica sólo considera en estado natural los indicadores socioeconómicos de la marginación, puesto que su intención no es fragmentar para después englobar, sino más bien, integrar para después analizar las partes agrupadas.

\section{Implicaciones socio-espaciales de la regionalización de las dimensiones de la marginación en Tamaulipas}

En este punto se aborda de manera analítica a través de sus indicadores de exclusión social, la clasificación regional por dimensión socioeconómica de la marginación en Tamaulipas. Como se especificó en el apartado metodológico, la técnica estadística empleada fue la de los clusters jerárquicos, que sirvieron para hacer las múltiples agrupaciones de acuerdo a sus respectivas distancias inter-grupos.

Esto se hace con la finalidad de identificar cómo a través de las reproducciones socioeconómicas puede vislumbrarse la marginación en cada una de ellas. Dichos grupos mantienen similitudes socio-espaciales e inclusive geográficas, así como en sus niveles de marginación.

Reconocer la importancia de las reproducciones socioeconómicas estriba en la elaboración de una política pública encaminada a las necesidades de la sociedad para superar los niveles de subdesarrollo y atraso representados por la marginación social, asimismo la política pública centrará, por orden de zonas prioritarias, las acciones regionales pertinentes para combatir los fenómenos de exclusión social, desigualdad, pobreza, etc., surgidos en el corazón del sistema económico y muy frecuentes en los países latinoamericanos.

Actualmente, existen espacios tanto dentro del país como en el estado de Tamaulipas que comparten muchas similitudes respecto a los diferentes grados de marginación aún sin pertenecer a la misma región geográfica, esto se debe a las diferentes reproducciones sociales y económicas que no ocurren solamente en una división política (regiones físicas de los municipios), es decir, pueden existir espacios con determinadas 
características similares en diferentes regiones geográficas, conformando otro espacio común, relacionado con una nueva geografía, la del espacio social.

De acuerdo a la lógica del estudio, la idea central sería ir identificando estos "espacios comunes" en donde converja el espacio social con el sociopolítico. Con base en lo anterior, surge la importancia de establecer regiones socioeconómicas para los diferentes aspectos estructurales de la marginación que estableció el CONAPO y que están representados por las diferentes variables de exclusión social y dimensiones socioeconómicas del índice de marginación, tal como se explicó en los puntos anteriores.

El estudio de las regiones sobrepasa la simple división política y la constitución geográfica debido a las diferentes interacciones sociales representadas por las variables sociodemográficas del índice de marginación.

Ahora bien, la dinámica de las interacciones sociales entre los diferentes actores que conforman el espacio permite el desarrollo del mismo, no como un simple territorio geográfico sino más bien constituye todo un complejo histórico, cultural, político y económico en torno a un sistema económico dado y a un imaginario colectivo.

En este caso, las desigualdades y reproducciones sociales son representadas por las dimensiones socioeconómicas de la marginación, es decir: educación, vivienda, ingresos y distribución poblacional, tales desigualdades constituyen no sólo carencias estructurales, sino también, como se ha mencionado a lo largo de este trabajo, diferencias sociales que inciden en la interacción tanto económica como simbólica de la población en sus espacios políticos, económicos y sociales.

Cabe mencionar que diferentes elementos como exclusión social, desigualdad y pobreza son formas de reproducción social muy particulares, como parte de la evolución del sistema económico en aras de la modernidad.

A partir de lo mencionado con anterioridad, la política social surge como respuesta inmediata a los cambios históricos del sistema económico que evoluciona la complejidad de la marginación.

Lo anterior se refiere a que la aglomeración del espacio social, representado por cada dimensión socioeconómica, constituye objetivamente grupos del espacio político para el logro de los fines políticos, es decir, refleja la "priorización de aglomeraciones espaciales" para la política pública y toda esta dinámica del Estado a través de los programas asistenciales y del gasto público, que impacta en el ingreso y consumo de la población en determinadas regiones.

Se entiende que la objetivación de la marginación tiene su reflejo en la intervención política, aunque también el diseño de otros espacios puede ser de gran utilidad para la instrumentalización de la política social y económica. Sin embargo, eso ya exige otro tipo de objetivos y planteamientos técnicos para su estudio.

Entonces, un mejor diseño e implementación de la política pública con base en la regionalización de la marginación, apoyará a la identificación de las zonas prioritarias por dimensión socioeconómica, mismas que se adecúan a la conformación del espacio social en el tiempo (en este caso para el 2005), en función de un constante flujo de elementos estructurales dentro de un modo de producción determinado, bajo la influencia de la globalización y el paradigma de la modernidad. 
A continuación se procede a mostrar las diferentes regiones (agrupaciones jerárquicas) de las dimensiones socioeconómicas de la marginación del estado de Tamaulipas, definido como un espacio político único compuesto de 43 municipios.

Cada inter grupo definido de los clusters estará clasificado por los grados de prioridad que coinciden, en gran parte, con los niveles de marginación de los municipios del estado, reflejando homogéneamente las diferencias en el espacio social dentro de las regiones.

Los grados de prioridad también serán de muy alta, alta, media, baja y muy baja, como en el caso del índice de marginación, sin embargo, esta graduación no pasó por una técnica de estratificación, debido a que los inter grupos definidos se hicieron de manera jerárquica, lo que significa que estarán distribuidos según la información de su varianza que coincide con sus índices de marginación, precisamente por esta agrupación homogénea.

La regionalización de la educación denota que la mayoría de la población ha tenido acceso a los servicios educativos, mejorando sus condiciones de alfabetismo y niveles de estudios. El siguiente cuadro describe lo aquí mencionado.

Cuadro I. Región-Educación

\begin{tabular}{|c|c|c|c|}
\hline Prioridad & Municipios (n) & $\%$ & \% Acumulado \\
\hline Muy alta & 2 & 4,65 & 4,65 \\
\hline Alta & 7 & 16,28 & 20,93 \\
\hline Media & 19 & 44,19 & 65,12 \\
\hline Baja & 9 & 20,93 & 86,05 \\
\hline Muy baja & 6 & 13,95 & 100,00 \\
\hline Total & 43 & 100,00 & \\
\hline
\end{tabular}

Fuente: Elaboración propia con datos de CONAPO, 2005.

De acuerdo a esta información, la mayoría de los municipios son de orden de prioridad media (19) que equivale a un $44,2 \%$, por lo que gran parte de la política pública debe concentrarse en una completa inclusión de estos municipios para que en los próximos años la dinámica económica y de las reproducciones sociales cambien y se adecúen a un desarrollo aproximado, ya que el elemento de la educación modificaría en buena manera su entorno espacial.

En municipios como Bustamante y Miquihuana urge el diseño de políticas educativas a nivel regional, debido a que el orden de prioridad es de muy alta; en menor medida, municipios como San Carlos, Tula o Casas deben ser atendidos.

Es importante mencionar que la dinámica social que se da entre los municipios de más a menos prioritarios también concuerda con sus grados de marginación desagregados, lo que involucra una desigualdad traducida a las diferenciaciones sociales que conforman un espacio social en función a las reproducciones sociales de las regiones. 
Esto sugiere que las condiciones de exclusión educativa de los municipios prioritarios espacialmente influyen en el desarrollo individual de sus habitantes, asociándolos más a las condiciones rurales, menores competencias laborales y resistencias hacia la inclusión a la modernidad, construyendo un tipo de vida social alejada de la integración social y económica.

La ventaja de vislumbrar la marginación desde su dimensión educativa es que permitirá la creación de estrategias de intervención social muy específicas para el combate de la pobreza y la disminución de la desigualdad, así como la gestión del gasto social en función de un criterio que identifica ordenadamente las necesidades específicas de la sociedad y los espacios tamaulipecos.

Esta información se puede representar gráficamente mejor en el siguiente mapa, en donde se observa la distribución del número de municipios por orden de prioridad en la política pública regional.

Figura 3. Marginación educativa por municipios y orden de prioridad.

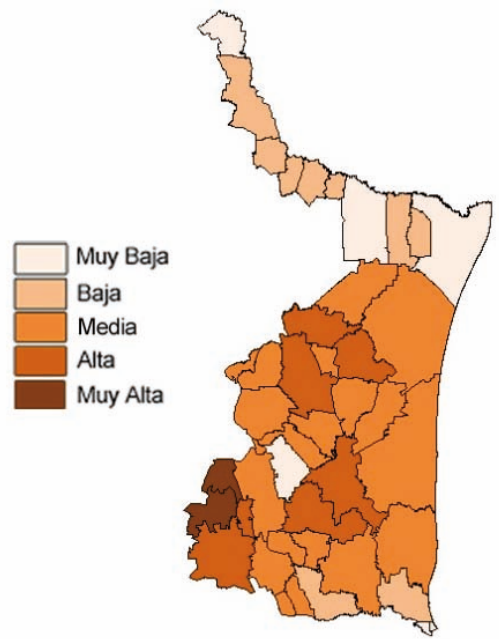

Fuente: Elaboración propia con datos del INEGI.

En lo que cabe, la evaluación de la marginación educativa es relativamente buena, al encontrar un grupo reducido de municipios de orden prioritario alto y muy alto. Sin embargo, el proceso de evaluación representa un diseño inteligente del largo plazo para poder mejorar estos indicadores.

Una vez diseñada la primera región, se procede a la presentación del siguiente cuadro, asociado con la dimensión vivienda, por ende, el aspecto de infraestructura y servicios públicos, el cual, su déficit representa a la marginación misma para el espacio tamaulipeco. 
Cuadro 2. Región-Vivienda

\begin{tabular}{|c|c|c|c|}
\hline Prioridad & Municipios (n) & $\%$ & \% Acumulado \\
\hline Muy alta & $\mathrm{I}$ & 2,33 & 2,33 \\
\hline Alta & 2 & 4,65 & 6,98 \\
\hline Media & 15 & 34,88 & 41,86 \\
\hline Baja & 8 & 18,60 & 60,47 \\
\hline Muy baja & 17 & 39,53 & 100,00 \\
\hline Total & 43 & 100,00 & \\
\hline
\end{tabular}

Fuente: Elaboración propia con datos del CONAPO, 2005

En materia de vivienda también se requiere mayor atención en un término medio, esto se puede deber a la incorporación de mayores servicios públicos hasta el 2005. Sin embargo esto no precisa que se haya mejorado del todo en relación con la vivienda, debido a que esta región se compone por la mayoría de los indicadores de la marginación (4), por lo que significa una relación promedio.

Sin embargo, el indicador cumple con su papel de mostrar una radiografía del estado en materia de infraestructura, señalando las condiciones generales de los municipios o estas carencias regionales.

Así, municipios como San Nicolás, Bustamante y Casas requieren mayor infraestructura, posteriormente la mayoría de los municipios también necesitarán el diseño de una política enfocada a sus necesidades específicas.

En menor medida, los municipios que tienen los niveles más bajos de marginación en el estado, como Tampico, Laredo, Cd. Madero, también los tienen en materia de vivienda, lo que denota un grado de desarrollo adecuado en su entorno espacial pero no homogéneo, de ahí la importancia nuevamente de continuar con ejercicios de mayor desagregación y regionalización espacial de la marginación. De la misma forma que en el primer caso, es conveniente ver esta distribución de los municipios por orden de prioridad, de manera gráfica. 
Figura 4. Marginación de vivienda por municipios y orden de prioridad.
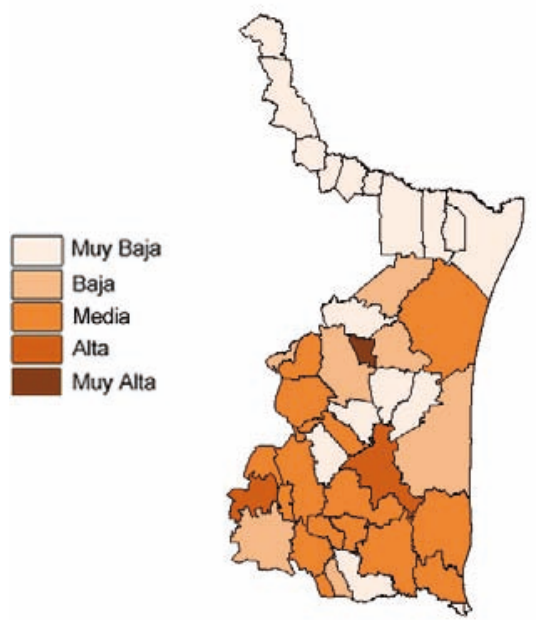

Fuente: Elaboración propia con datos del CONAPO, 2005.

Aunque la dimensión vivienda parezca ser de índole meramente material por tratarse de infraestructura regional, no quiere decir que exima a los componentes culturales y sociales de las dinámicas a nivel municipal del modelo desagregado de la marginación.

La política en esta materia aparte de dotar de infraestructura de calidad a los municipios debe reconocer la reproducción social de esta dimensión: el hecho de que existan carencias de vivienda a nivel municipal quiere decir que hay una dinámica de diferencias sociales, puesto que el porcentaje de la población por ejemplo que no cuentan con sanitario exclusivo y drenaje recrea otra dinámica espacial de grupos, respecto al porcentaje de la población que sí cuenta con ese servicio.

En estos términos de relaciones sociales está en juego la consideración de la automarginación, donde la cultura se antepone a la concepción del desarrollo en infraestructura de la vivienda, debido a la resistencia hacia la modernidad.

Entonces toda política pública, a través de las regiones de prioridad del espacio social, debe poner atención a las reproducciones sociales de los municipios con mayores carencias en infraestructura de vivienda y no sólo tener una visión unilateral de la dotación de servicios modernos, debido a que se tiende a la exclusión del entorno cultural e histórico.

La política social en materia de vivienda debe ser multidiversa y cada vez más específica, de acuerdo a una consideración holística de los espacios sociales, porque en regiones atrasadas económicamente o no desarrolladas se genera una mayor resistencia en el entorno con la implementación de alguna intervención del Estado, ya sea federal o estatal. 
Conviene señalar ahora el factor de la región asociada a la dimensión de los ingresos, un aspecto muy importante de la marginación puesto que con base en éste se deriva una porción del bienestar, funcionando también como una herramienta fundamental para el acceso a bienes y servicios considerados parte del desarrollo social.

No obstante, eso no significa que sea totalmente determinante en las interacciones sociales, pero al menos sí es muy importante, ya que involucra otros elementos de orden sociológico, político y cultural.

Cuadro 3. Región-Ingresos

\begin{tabular}{|c|c|c|c|}
\hline Prioridad & Municipios (n) & $\%$ & \% Acumulado \\
\hline Muy alta & 4 & 9,30 & 9,30 \\
\hline Alta & 13 & 30,23 & 39,53 \\
\hline Media & II & 25,58 & 65,12 \\
\hline Baja & 8 & 18,60 & 83,72 \\
\hline Muy baja & 7 & 16,28 & 100,00 \\
\hline Total & 43 & 100,00 & \\
\hline
\end{tabular}

Fuente: Elaboración propia con datos de CONAPO, 2005.

En ingresos la situación cambia, aproximadamente un $40 \%$ de los municipios se concentran en un orden de prioridad alto y muy alto, un $25,5 \%$ en un término medio y un $34,5 \%$ en el estrato bajo y muy bajo.

La reproducción, relación o diferencia social del ingreso en el espacio dictaminará un patrón de estilo de desarrollo muy específico, con determinadas influencias de la modernidad en esta interacción global consciente o inconsciente entre los individuos. La marginación en ingresos atiende al subdesarrollo y a la desigualdad de la distribución del ingreso nacional, ahora regional.

En el gráfico anterior se observan desigualdades regionales muy remarcadas, lo que agudiza las problemáticas de corte estructural en el estado. Toda política social debe orientarse a la generación de condiciones adecuadas para un desenvolvimiento socio espacial que promueva el desarrollo económico y social, comenzando por las zonas más vulnerables.

Municipios como Bustamante, Casas, Palmillas y San Nicolás requieren una incorporación inmediata a las esferas productivas para poder afrontar la vulnerabilidad a la que están sujetos.

A otros como Jaumave, Miquihuana, Tula y San Carlos, en menor medida pero no careciendo de importancia, deben planteárseles mecanismos de producción y distribución para la mejora del empleo y la explotación racional de los recursos.

El siguiente mapa refleja la información aquí presentada: 
Figura 5. Marginación de ingresos por municipios y orden de prioridad.
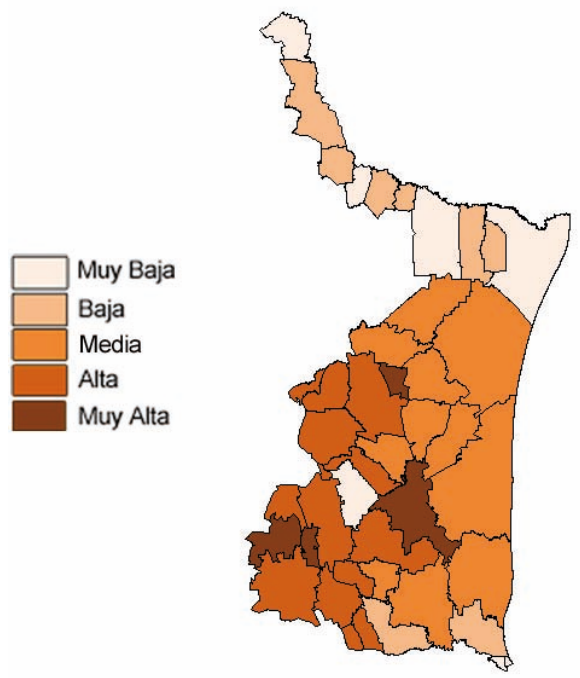

Fuente: Elaboración propia con datos del CONAPO, 2005.

Gran parte de la población en el estado de Tamaulipas ingresa no más de dos salarios mínimos, los que no son capaces de abastecer los requerimientos básicos de la población en los municipios, debido a las presiones inflacionarias y la volatilidad de la actividad económica en momentos de recesión y crisis.

A lo anterior habría que agregar las políticas neoliberales de bajo costo de la mano de obra que generan presiones para los diferentes hogares con ese nivel de ingresos, lo que provoca reacciones socioeconómicas en el espacio social en materia de ingresos en el estado.

La política social sabrá entonces hacia dónde enfocar la política económica de una manera más específica. Aunque una política equitativa e incluyente debe esforzarse por localizar las microrregiones que reflejen las condiciones carenciales en el ingreso, aún en los municipios de menor prioridad, porque los niveles de prioridad a nivel localidad y conglomerados más pequeños tenderán a modificarse entre más se relocalicen estas carencias o entre más subespacios sociales surjan de sus respectivas microrregiones.

Sin duda, la dimensión de los ingresos no sólo consiste en las remuneraciones suficientes para el consumo de bienes y servicios, también se asocia al rubro de la renta regional dentro de los municipios, en donde intervienen aspectos relacionados con la capacidad productiva de la población.

Por lo que la dimensión económica de la marginación no sólo consiste en el ingreso mínimo, sino también en las potencialidades productivas mínimas del individuo y sus diferentes grupos, así como en sus niveles de incorporación a la actividad 
económica, sus condiciones espaciales de movilidad forzada (migración por cuestiones laborales), la durabilidad del trabajo, la informalidad del trabajo, etc.

Se trata de elementos de coyuntura que conformen la súper población relativa tanto preparada y no preparada escolarmente y que incidan en esta conformación de la geografía social de las carencias (economía) y las diferencias (sociedad-cultura).

El aumento del crecimiento económico en las regiones se ve influenciado por la concentración de la actividad económica, sobre todo aquella que, por medio de la tecnología y la especialización, generan valor agregado a los productos.

En otras palabras, los niveles de concentración industrial y de tecnologización y especialización en los municipios serán la plataforma fundamental de las condiciones económicas para reactivar el capital regional y generar una dinámica que repercuta en los niveles de ingresos y bienestar en los espacios interestatales.

Por último corresponde presentar a la región asociada con la distribución de la población, este factor expone una radiografía de la situación urbana y rural del estado visto como un único espacio y sus respectivos espacios sociales.

Cuadro 4. Región-Distribución de la población

\begin{tabular}{|c|c|c|c|}
\hline Prioridad & Municipios (n) & $\%$ & \% Acumulado \\
\hline Muy alta & 19 & 44,19 & 44,19 \\
\hline Alta & $\mathrm{n} 2$ & 4,65 & 48,84 \\
\hline Media & 8 & 18,60 & 67,44 \\
\hline Baja & 5 & 11,63 & 79,07 \\
\hline Muy baja & 9 & 20,93 & 100,00 \\
\hline Total & 43 & 100,00 & \\
\hline
\end{tabular}

Fuente: Elaboración propia con datos del CONAPO, 2005.

De acuerdo al cuadro anterior, esta es la región que contiene los municipios de mayor orden de prioridad. Más del $49 \%$ está en condiciones de prioridad muy alta y alta, el $19 \%$ se distribuye en el nivel medio y un 32\% en los niveles bajo y muy bajo.

Obsérvese que la distribución de la población está polarizada, es decir, por una parte son rurales y otros urbanos, y otra gran parte se encuentra en un proceso de transición o cambio hacia lo urbano. Esto demuestra la tendencia hacia un alto crecimiento de la urbanización en los espacios económicos y sociales, desplazando poco a poco la vida rural del país y sus regiones.

Esta región se puede apreciar de mejor manera a través del siguiente mapa que muestra la distribución de los municipios en función a la marginación poblacional por orden de prioridad para la atención social. 
Figura 5. Marginación por distribución de la población de los municipios y su orden de prioridad.
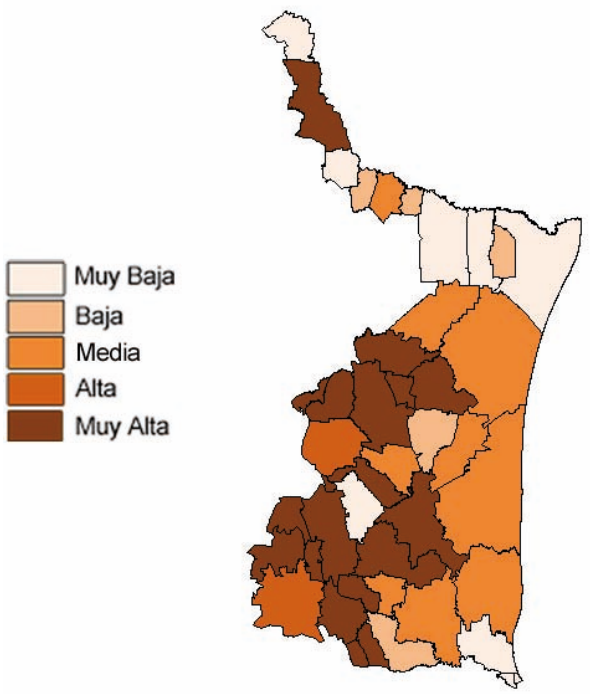

Fuente: Elaboración propia con datos del CONAPO, 2005.

Municipios como San Nicolás, Casas, Bustamante y Palmillas, mantienen una dinámica social meramente rural, continuados por otros, como Llera, Miquihuana, Tula, San Carlos, Ocampo, Jaumave, etc.

Lo anterior no quiere decir que todas las localidades de los municipios estén totalmente desurbanizadas, sólo se muestra una generalización espacial de la población, que en estos municipios es de características rurales. Los municipios que se encuentran en un proceso difícil de transición, por citar solo algunos, son San Fernando, Soto la Marina,Aldama, Abasolo y González.

Por el contrario, los que mantienen un ritmo alto de urbanización son los municipios de: Tampico, Reynosa, Cd. Madero, Matamoros y Laredo, los cuales representan una evolución impresionante en la urbanización del espacio económico y social, debido a que también son centros de desarrollo industrial y comercial, lo que obliga a una rápida movilidad social de las regiones o municipios aledaños, como esfuerzo de la integración a las actividades económicas, asociadas mayormente con el pleno empleo.

Nótese entonces que en el caso de Tamaulipas hay una gran desigualdad en los sectores urbanos y rurales. Los primeros enfrentan movilidades sociales y concentración de la marginación en las periferias debido a que se han convertido en polos de desarrollo económicos muy importantes; los segundos denotan una mayor vulnerabilidad por la reducción de su población, condiciones geográficas adversas asociadas con el clima (seco en la mayoría del territorio) que impide el logro de una producción del campo suficiente, así como la obstaculización al acceso para la implementación de 
servicios públicos tanto a las viviendas como a las localidades, por ejemplo, las vías de comunicaciones y transporte.

No es la intención denotar que los municipios de menor prioridad asociados a lo urbano sean menos marginados por el hecho de ser urbanos, o por el contrario, más marginados por el hecho de ser rurales.

La idea central hace hincapié en que, efectivamente, la marginación en Tamaulipas se asocia al sector rural, debido a la homogenización del estilo de desarrollo y de otras características convergentes en las relaciones de producción, de reproducción social y geográfica, a diferencia de los espacios urbanos, donde hay más heterogeneidad de estas características socioeconómicas, lo que provoca una mayor complejidad de análisis.

Lo que sí es importante es que esta regionalización de la dimensión de distribución poblacional puede dividir claramente los municipios de mayor ruralidad y urbanidad para la planeación sectorial de la política pública regional; es decir, permite ubicar necesidades de acuerdo a las condiciones poblacionales de los espacios. También contribuye a la discusión de una planificación territorial incluyente y sustentable en todo momento.

No debe olvidarse que esta relación de lo rural con lo urbano también se refiere a la reproducción de la sociedad histórica y la moderna, habiendo un flujo de intercambio de diferencias regionales en los municipios, trasladando símbolos que se combinan con el tipo de desarrollo económico y sus desequilibrios producto del juego de tensiones de las fuerzas del mercado regional y global que afecta a toda la población y sus territorios.

Una política regional incluyente conviene que reconozca que los procesos de modernización en los municipios son paulatinos y muy complejos, por lo que acondicionar las regiones menos modernas bajo un criterio de respeto a sus reproducciones sociales es concerniente impulsar este cambio desde la aceleración de la economía regional y bajo el apoyo de programas locales de acuerdo a la prioridad política acerca de la enseñanza de la modernidad.

Asimismo este espacio social previene el orden de planificación de los municipios, de acuerdo a sus tasas de urbanización e identifica aquellos espacios de letargo de los cambios estructurales hacia la modernidad, que generan también diferencias.

Con esto se comprende que aunque todos los espacios fueran urbanos, seguirá habiendo una resistencia en microespacios hacia la modernidad, puesto que este concepto es un estilo de desarrollo adaptado e inclusive impuesto, que en términos constantes el tipo de desarrollo histórico y cultural no siempre lo acepta, reproduciendo nuevamente las diferencias sociales, por lo que la política en esta materia debe tener muy en cuenta estas condiciones.

Hasta aquí se expusieron las cuatro regiones que representan a cada una de las dimensiones de la marginación por dimensión socioeconómica y los componentes de exclusión social para el estado de Tamaulipas, logrando hacer observaciones pertinentes en materia de política pública regional. 
Se abre la discusión para la implementación de modelos locales en la toma de decisiones de los diferentes niveles de gobierno en un marco de descentralización, democratización de los actores sociales y la globalización, de acuerdo a las diferentes interacciones que forman el espacio no sólo político, sino también el social.

De esta forma se determinó la fisonomía de una geografía social de las carencias y de las diferenciaciones de acuerdo a la reflexión sobre el espacio social de Bourdieu y su relación con la política pública en los diferentes procesos de reconfiguración por dimensión socioeconómica de la marginación.

\section{Conclusiones}

El análisis que se hizo sobre las determinaciones de regiones socioeconómicas por dimensión de la marginación sugiere que cada dimensión analizada contenga una serie de diferenciaciones muy particulares, que definen distancias socioeconómicas de la población en sus respectivos espacios.

En este caso, los municipios del estado irán constituyendo un espacio social muy particular, puesto que están regionalizados en función a las dimensiones socioeconómicas de la marginación; lo aquí mencionado indica que se establece una serie de reproducciones sociales muy específicas entre la población y sus dinámicas sociales y contextuales, determinando límites sociales y simbólicos, es decir, límites de diferenciación.

Por ejemplo, de una forma integral (agregada) el municipio más marginado es el de Bustamante, sin embargo al analizar sólo la dimensión de educación, el municipio de San Nicolás es el que presenta el mayor grado de exclusión en esta categoría y el posicionamiento del municipio a nivel inter estatal le otorga otra tesitura geográfica y socioeconómica en sus reproducciones sociales por ser de los municipios más excluidos y en donde su condición sobre esta dimensión también es influyente para el desarrollo de la vida social.

Esta combinación entre la técnica de cluster y las aplicaciones teóricas de Bourdieu respecto al espacio social, complementan la comprensión de la marginación desde perspectivas cada vez más regionales.

Lo anterior toma en cuenta aspectos relacionados a lo simbólico y real de las reproducciones sociales reflejadas en la estructuralidad socioeconómica, que es diferenciable (falta de acceso a bienes y servicios que ofrece el desarrollo económico) entre los distintos grupos poblacionales pertenecientes a espacios políticos (en este caso municipios).

De esta forma se constituye la geografía del espacio social de la marginación, que puede ser de gran ayuda para el diseño y la implementación de políticas públicas municipales más eficientes que relocalicen las problemáticas sociales, económicas y culturales de las desigualdades regionales.

La nueva política social debe considerar el criterio regional para la toma de decisiones, reconociendo que son las diferentes relaciones socio-espaciales de la po- 
blación, dentro de un contexto globalizador, las que determinan las intensidades socioeconómicas del territorio.

Lo aquí planteado pertenece a la marginación regional enfocada a los grupos donde intervienen elementos culturales, históricos y espaciales, y la acción pública, a través de la política social, la cual juega un papel importante para la inclusión de estos sectores en sus respectivas geografías.

\section{Referencias}

Bourdieu, P. et.ál. (1993). La misere du monde. París: Du Seuil.

Bourdieu, P. (2002). “Espacio social y espacio simbólico”. Razones Prácticas: Sobre la teoría de la acción. $3^{\mathrm{a}}$ ed. Barcelona:Anagrama, pp. I I-26.

Campoy, M. (2002). “Marginación y pobreza”. Revista del Ministerio del Trabajo y asuntos sociales, (35), Pp. 67-82. En: <http://dialnet.unirioja.es/servlet/ oaiart?codigo $=253634>$.

Cogco, A. (2010). “Discusión teórica de la marginación”. Reporte de investigación (no publicado). Universidad Autónoma de Tamaulipas/Fondos Mixtos.

Conapo. (1993). Indicadores socioeconómicos e índice de marginación municipal, 1990. México: Conapo/Cna.

Conapo. (2000). Metodología para el cálculo de la marginación, 2000. México: Conapo.

Conapo. (2005). “Anexo C: Metodología de estimación del índice de marginación para el 2005”. En: <http://www.conapo.gob.mx/publicaciones/margina2005/ AnexoC.pdf>.

Conapo. (2005). Índice de marginación a nivel localidad. En <www.conapo.gob.mx/publicaciones/IndiceMargLoc2005.pdf>.

Conapo-Progresa. (1999). Índices de marginación, 1995. México: Conapo.

Coplamar-Presidencia de la República. (1977). Bases para la acción 1977-1982. México: Coplamar-Palacio Nacional.

Coplamar-Presidencia de la República. (1979). Mínimos de bienestar. México: Coplamar.

Coplamar. (198I). Macroeconomía de las necesidades esenciales en México. México: Siglo $\mathrm{XXI}$ editores.

Cortés, F. (2002). “Consideraciones sobre la marginalidad, marginación, pobreza y desigualdad en la distribución del ingreso”. Papeles de población, (31), pp. 9-24. En: <http://redalyc.uaemex.mx/redalyc/pdf/ I I / I I 204704.pdf>.

Cortés, F. (2006). “Consideraciones sobre la marginación, marginalidad, marginalidad económica y exclusión social”. Papeles de población, (47), pp. 7I-84. En: <http:// redalyc.uaemex.mx/redalyc/src/inicio/ArtPdfRed.jsp?iCve= I | 204704>.

Galvis, J.P. (2002). “La dimensión urbana de la marginalidad en la Orinoquia”. Tres dinámicas diferentes de su reproducción, (7), pp. 89-107. En: <http://redalyc.uaemex. $\mathrm{mx} / \mathrm{redalyc} / \mathrm{html} / 290 / 2900$ | 9 |2/2900|9|2.html>.

Gutiérrez,A. (2003). "La construcción social de la pobreza: un análisis desde las categorías de Bourdieu”. Anduli (2), pp. 28-44.

Otero, E. C. (1997). Construcción de un índice de marginación por localidad-del concepto al indicador-desigualdad económica y social de las localidades de México en 1990. UNAM:Acatlán. 
Noda, E. (2010). La espacialidad de la marginación en el estado de Tamaulipas y sus implicaciones cuantitativas de la desagregación y regionalización socio espacial. Universidad Autónoma de Tamaulipas, México.

Otero, E. C. (2003). "¿Qué miden el índice de marginación y el índice de desarrollo humano?". Estudio de caso, municipios de México, 2000. Facultad Latinoamericana de Ciencias Sociales, México.

Terrádez, M. 2010. Dendograma: En: <http://www.uoc.edu/in3/emath/docs/Cluster. $p d f>$.

Villardón, V. 2010. Dendograma. En: <http://benjamindespensa.tripod.com/spss/ AC.pdf>.

Visauta B. y Martori, J. (2003). Análisis estadístico con SPSS para Windows: Estadística multivariable ( $2^{\mathrm{a}}$ ed., vol. 2). Madrid: Mc Graw Hill. 